\title{
LA VULNERABILIDAD FISCAL DEL GASTO SOCIAL: ¿ES DIFERENTE AMERICA LATINA?
}

THE FISCAL VULNERABILITY OF PUBLIC SOCIAL EXPENDITURES: IS LATIN AMERICA DIFFERENT?

\section{EDUARDO LORA*}

Banco Interamericano de Desarrollo

\begin{abstract}
An unbalanced panel data of around fifty countries between 1985 and 2003 is used to estimate the vulnerability of public social expenditures (health and education) to other fiscal variables. The database allows comparisons between Latin America and the rest of developing countries. Public social expenditure is significantly lower in Latin America as share of GDP, although it has a higher share in primary expenditure. Public social expenditures in Latin America are more vulnerable to debt service, but are less sensible to changes in other types of public expenditure. As in other regions in the developing world, public social expenditure in Latin America shrinks when public debt stock increases. This effect is higher with multilateral debt obligations. In Latin America, debt defaults reduce the share of public social expenditures in total primary public expenditure.
\end{abstract}

Keywords: Public Social Expenditure, Public Debt, Latin America, Primary Expenditures, Debt Services, Multilateral Debt, Defaults.

JEL Classification: H51; H52; H63; F34; F53.

* Gerente del Departamento de Investigación y Economista Jefe (a.i.), Banco Interamericano de Desarrollo. 1350 New York Avenue NW, Washington D.C., U.S.A. Tel: +1 (202) 623 1271. Fax: +1 (202) 623 1772. Email: eduardol@ iadb.org. El autor agradece la valiosa ayuda de Juan Camilo Chaparro y Lucas Higuera y los comentarios de Eduardo Borensztein, Ugo Panizza y un revisor anónimo de la Revista de Análisis Económico. 


\section{Resumen}

Se utiliza un panel desbalanceado de medio centenar de países para el período 1985-2003 con el objeto de evaluar la vulnerabilidad del gasto público social (en educación y salud) frente a las variables fiscales corrientes y la deuda pública en América Latina en comparación con el resto del mundo en desarrollo. El gasto social es significativamente más bajo en América Latina (aunque absorbe una proporción mayor del gasto primario) y es más vulnerable frente a mayores pagos de intereses de la deuda, pero más insensible a las variaciones del resto del gasto público. Como en otras regiones del mundo en desarrollo, el gasto social en América Latina se contrae con los aumentos de deuda pública, y aún más si se trata de deudas con la banca multilateral. A diferencia del resto del mundo, en América Latina los incumplimientos de deuda llevan a reducir la participación del gasto social en el gasto público.

Palabras Clave: Gasto Público Social, Deuda Pública, América Latina, Gasto Primario, Intereses sobre la Deuda, Deuda Multilateral, Default de la Deuda.

Clasificación JEL: H51; H52; H63; F34; F53.

\section{INTRODUCCION}

En contra de la opinión corriente, el gasto social ha aumentado en América Latina desde la adopción de las políticas del Consenso de Washington: entre 1990 y 2003 el gasto social por habitante en toda la región creció 58\% (a precios constantes) y como proporción del PIB pasó de 9\% a 11\%. ${ }^{1}$ Sin embargo, los latinoamericanos consideran que los gobiernos deberían dedicar más recursos a la salud, la educación básica y la seguridad social ${ }^{2} \mathrm{y}$, a juzgar por la visibilidad del tema en las campañas electorales de los últimos años, demandan políticamente que los gobiernos adopten medidas concretas para hacerlo. Las políticas que propusieron como candidatos varios de los actuales presidentes cubren todo el espectro posible, desde redoblar la disciplina fiscal y reorientar el gasto hacia las áreas sociales, como lo prometieron Alan García en Perú y Luiz Inácio (Lula) da Silva en Brasil, hasta dejar de pagar las deudas públicas, como lo anunció entonces y lo repite cada cierto tiempo Rafael Correa en Ecuador, pasando por generar mayores recaudos tributarios con destino social, como una y otra vez lo ha intentado Alvaro Uribe en Colombia. Tras todas estas opciones

1 Según datos de la CEPAL (2007) que incluyen educación, salud, seguridad social y vivienda, y cubren 18 países.

2 Según el Latinobarómetro para 1998, los latinoamericanos piensan que el Estado debería gastar más en salud (92\% de los encuestados), en educación básica (57\%) y en seguridad social (75\%). 
está la presunción de que el gasto social es vulnerable ante eventuales reveses de la situación fiscal y ante las políticas hacia las que se inclinan los ministros de finanzas para cumplir las obligaciones crediticias o para ajustar las cuentas públicas cuando es necesario hacerlo.

Aunque la vulnerabilidad del gasto social frente a las políticas de ajuste fiscal y de endeudamiento público ocupa un lugar prominente en el debate político, ha atraído muy poca atención académica. Desde los años ochenta unos pocos estudios han sugerido que los gastos sociales son más resistentes que otros gastos en los períodos de ajuste fiscal. Por ejemplo, Hicks y Kubisch (1984) y Hicks (1989) encontraron que en los países altamente endeudados, los ajustes fiscales de la década del setenta y comienzos de los ochenta afectaron muy poco al gasto social. Baqir (2002) llegó esencialmente a la misma conclusión con un panel de más de 100 países para el período 1985-1998. ${ }^{3}$ En una vertiente de estudios relacionada con este tema se ha encontrado que las categorías de gasto más sensibles a los ajustes fiscales no son las más próximas al gasto social. Heller y Diamond (1990), con una muestra amplia de países para el período 1975-1986 encontraron que el cambio más común en los patrones de gasto cuando hay ajustes fiscales es en contra de los gastos de capital fijo y las transferencias de capital y a favor de los pagos de interés, los subsidios y las transferencias. De forma semejante, Papagapitos (1992), con una muestra de 25 países para el período 1972-1988, concluyó que los programas de estabilización no desplazan el gasto público en contra de las "áreas pro crecimiento", las cuales incluyen los gastos en educación y salud.

Un pequeño grupo de estudios ha analizado los efectos del endeudamiento sobre el gasto social. Mahdavi (2004) intentó medir el impacto de la carga de las deudas externas sobre la composición del gasto público por categorías económicas. Con una muestra de 47 países para el período 1972-2001, encontró que la carga de la deuda reduce el gasto de capital y los gastos corrientes diferentes de sueldos y salarios. Puesto que gran parte del gasto social toma la forma de pagos salariales a los empleados públicos de la salud y la educación, este resultado sugiere que el gasto social está relativamente protegido de los efectos adversos del endeudamiento. Sin embargo, como se argumenta en Lora y Olivera (2007), esta conclusión puede ser engañosa porque parte de los gastos sociales no son sueldos y salarios, y porque el endeudamiento no es solo externo. De hecho, cuando se toma en cuenta la totalidad de la deuda pública y una definición apropiada del gasto social se encuentra que éste sí es vulnerable a los aumentos de la deuda y a los mayores pagos de intereses de la deuda. El estudio de Lora y Olivera también analizó el efecto de los incumplimientos de la deuda (defaults), uno de los temas más polémicos en el debate público latinoamericano, llegando a la conclusión de que, en el corto plazo, sí contribuyen a elevar el gasto social. Finalmente, la eficacia de los alivios de deuda como instrumento para elevar el gasto social ha sido estudiada por Chauvin y Kraay (2005). Utilizando cálculos del valor presente de los alivios de deuda en 62 países de bajos ingresos entre 1989 y 2003 ,

3 El principal objetivo de este estudio era analizar la influencia de variables políticas e institucionales en el gasto social. Otros estudios que han abordado este tema son Mauro (1998) y Snyder y Yackovlev (2000). 
han concluido que "hay poca evidencia de que los alivios de deuda hayan afectado el nivel o la composición del gasto público en los países beneficiarios".

Esta breve reseña de literatura muestra que la sensibilidad del gasto social ante las políticas fiscales ha sido un tema poco analizado. De la mayoría de estudios sólo pueden hacerse inferencias indirectas sobre el gasto social, ya que no utilizan medidas directas de esta variable. Unos pocos estudios recientes que sí utilizan información de gastos sociales se han concentrado en sus relaciones con la deuda, especialmente externa, pero ni unos ni otros se han ocupado expresamente de establecer si las conclusiones son extensivas a América Latina.

Este artículo intenta dar respuesta a las siguientes preguntas centrales para entender las reacciones del gasto social frente a las variables fiscales y de endeudamiento (precisando en cada caso las diferencias entre los países latinoamericanos y el resto del mundo en desarrollo):

- ¿Cómo responde el gasto social a los cambios en el gasto público primario (sin pagos de intereses de la deuda), a los cambios en los ingresos fiscales, y a variaciones en los pagos de intereses y en los saldos de la deuda pública (interna y externa)?

- ¿Cómo difieren estas respuestas para los gastos en educación y en salud?

- ¿Existe alguna diferencia en el comportamiento del gasto social si los gobiernos se financian con fuentes oficiales externas (banca multilateral, banca bilateral y Fondo Monetario Internacional)?

- ¿Qué ocurre con el gasto social cuando los países incumplen sus obligaciones de pago de las deudas?

Las respuestas a algunas de estas preguntas son sorprendentes. El gasto social responde mucho más fuertemente a los aumentos de los gastos primarios que a los aumentos de los ingresos fiscales y por lo tanto es muy sensible a la combinación de políticas que se utilicen en los períodos de ajuste fiscal. En el extremo, una política de ajuste basada enteramente en mayores impuestos llevaría a aumentar el gasto social. Sin embargo, en América Latina — no así en el resto del mundo- el gasto social resulta desplazado por los mayores pagos de intereses de la deuda. Aparte de este efecto, mayores saldos de deuda pública reducen el gasto social, tanto en la región como en el resto del mundo. El endeudamiento con la banca oficial no mitiga este efecto, antes bien parece aumentarlo, posiblemente porque contribuye a disciplinar el gasto total. Los incumplimientos de las obligaciones crediticias en el resto del mundo pueden tener algún efecto favorable sobre el gasto social (más exactamente sobre su participación en el gasto total), pero no en América Latina, donde generan el efecto contrario (excepto en países con niveles extremadamente elevados de deuda).

Las implicaciones de política de estos resultados son bastante directas: en América Latina, donde los gastos sociales son muy bajos en nivel, pero muy altos como proporción del gasto total, es preciso elevar los recaudos tributarios para poder elevarlos. Los gobiernos de la región deben moderar el endeudamiento puesto que su efecto sobre el gasto social es negativo tanto en forma directa como, sobre todo, a través de los mayores pagos de intereses. Excepto países en situaciones de sobreendeudamiento 
extremo, los demás protegen mejor el gasto social cumpliendo con sus obligaciones crediticias que rechazándolas.

\section{BASES DE DATOS Y ESTRATEGIA ECONOMETRICA}

Nuestra base de datos es un panel de datos anuales para el período 1985-2003 que cubre hasta 58 países en desarrollo (la muestra en cada regresión oscila entre 40 y 57 países debido a información incompleta). La información sobre gastos sociales proviene de una base de datos compilada por el Departamento de Asuntos Fiscales del Fondo Monetario Internacional. ${ }^{4}$ Como lo explica Baqir (2002), estos datos son más confiables que la base más conocida del Government Finance Statistics (GFS), porque la información se toma directamente de los documentos producidos para cada país por el FMI. La definición de gobierno utilizada puede diferir en distintos países, siendo gobierno central en unos y gobierno general en otros, pero el tratamiento es consistente en el tiempo en cada país. Los gastos sociales considerados incluyen solo educación y salud y se presentan como proporción del PIB nominal o como proporción de los gastos primarios del gobierno (es decir, sin incluir pago de intereses), utilizando información del International Finance Statistics (IFS) del Fondo Monetario Internacional y del GFS, respectivamente. ${ }^{5}$

La información sobre todas las variables fiscales, excepto deuda, es tomada del GFS. Puesto que esta información viene expresada en valores nominales, se convierte a proporciones del PIB utilizando los valores del PIB corriente reportados en el IFS. Los datos de deuda pública provienen de una nueva base de datos preparada por el Banco Interamericano de Desarrollo, BID, con base en información del IFS, complementada con datos del World Economic Outlook del FMI y de la CEPAL, principalmente. ${ }^{6}$ A diferencia de otras bases de datos sobre deuda pública, ésta incluye tanto la deuda externa como la interna de los gobiernos centrales (aunque no de otros niveles de gobierno o de las empresas estatales). Los valores de la deuda en monedas extranjeras se convierten a monedas nacionales usando las tasas de cambio de mercado. ${ }^{7}$ Finalmente, la variable dummy de incumplimiento de las obligaciones de deuda (que toma el valor de 1 en los años en que el país está en mora) proviene de datos de Standard \& Poor's procesados por Borensztein y Panizza (2006). El Cuadro 1 presenta las estadísticas principales de las variables utilizadas.

El principal problema que debe tenerse en cuenta en las estimaciones es el sesgo de endogeneidad que resultaría de estimar directamente el gasto social en función

4 Esta base de datos se describe en detalle en Gupta y otros (2000) y es la misma utilizada en Lora y Olivera (2007) y en Baqir (2002), en este último artículo con información hasta 1998.

5 Existe también la información de gasto social que recopila la CEPAL con información obtenida directamente de los gobiernos y procesada en forma estandarizada según las propias definiciones de esa entidad. Debido al objeto de este artículo, no utilizamos esta base puesto que solo cubre América Latina. Sin embargo, hemos comprobado que ambas bases son muy semejantes para los países comunes.

6 Para una descripción detallada véase Jaimovich y Panizza (2006) y BID (2007).

7 Para evitar que los resultados queden dominados por observaciones extremas de esta variable, se han excluido de la muestra las observaciones en que la deuda como proporción del PIB supera el $150 \%$. 


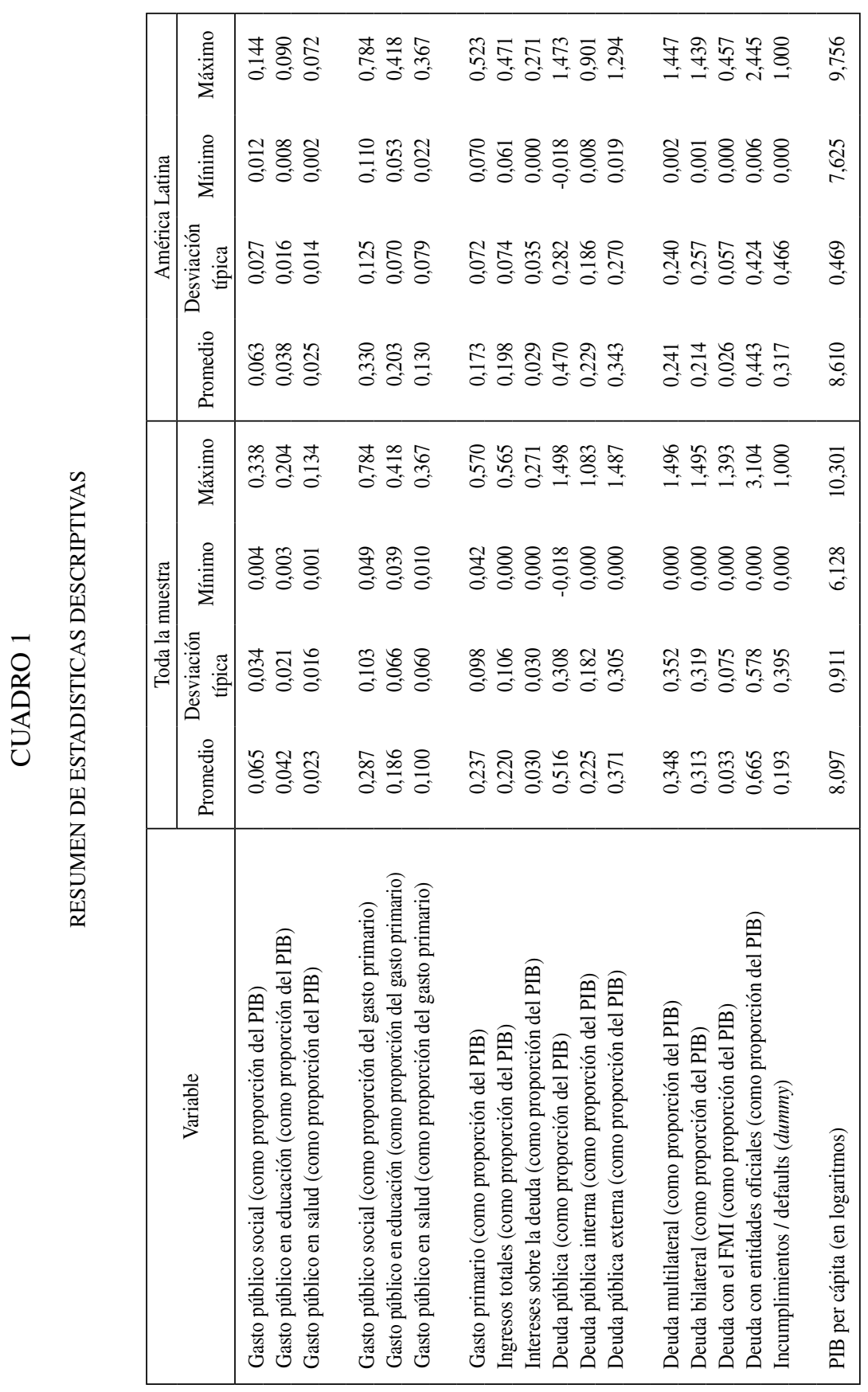


de las variables fiscales. El método más conveniente para evitar la endogeneidad en regresiones de panel en el sistema de estimación de Arellano y Bond (1991), el cual utiliza valores rezagados de las variables explicativas (en primeras diferencias) como instrumentos de esas mismas variables. La validez del método descansa en el supuesto de que los instrumentos estén correlacionados con las variables explicativas pero no con la variable dependiente. ${ }^{8}$ La prueba de Sargan (que se reporta en las principales regresiones en el Cuadro 3) es un criterio sencillo para saber si el supuesto tiene validez, como es el caso en la mayoría de nuestros resultados. ${ }^{9}$ Puesto que en el método de Arellano y Bond la variable dependiente se expresa en primeras diferencias, queda obviado el problema potencial de no estacionaridad que suelen exhibir las series en niveles, y que podría resultar en correlaciones espurias. ${ }^{10}$

Puesto que el objetivo central de este artículo es analizar si en América Latina los gastos sociales son más sensibles a las variables fiscales que en el resto del mundo en desarrollo, las regresiones incluyen en adición a las variables fiscales explicativas estas mismas variables multiplicadas por una variable dummy para los países latinoamericanos. Los coeficientes de estas últimas variables reflejan directamente si América Latina es diferente en cada una de las dimensiones consideradas.

\section{LAS REACCIONES DEL GASTO SOCIAL A LAS VARIABLES FISCALES}

América Latina suele asociarse con inestabilidad fiscal y macroeconómica. Puesto que, como veremos en seguida, el gasto social en la región está por debajo de los patrones mundiales para los países en desarrollo, es natural preguntarse si la respuesta del gasto social a las diversas variables fiscales es diferente en América Latina.

Como porcentaje del PIB, los gastos sociales en la región están 1,7 puntos por debajo del patrón internacional (Cuadro 2, regresión 1). La brecha es significativa al $1 \%$, y ha sido calculada después de controlar por el nivel de ingreso per cápita y su cuadrado (aunque estos controles no son significativos). ${ }^{11} \mathrm{El}$ grueso de la brecha se debe al sector de educación, donde la diferencia promedio entre América Latina y el resto del mundo en desarrollo es 1,2 puntos del PIB. Sin embargo, cuando el análisis se hace no con proporciones del PIB sino con proporciones del gasto público primario, resulta que el gasto social en América Latina es mayor que en el resto del mundo en

8 Si los instrumentos son débiles, los coeficientes estimados por este método pueden ser inconsistentes, caso en el cual puede ser más adecuado utilizar el método de momentos generalizado con variables instrumentales GMM-IV desarrollado por Arellano y Bover (1995) y por Blundell y Bond (1998), en el cual se incorporan en la especificación los niveles y las primeras diferencias de la ecuación en un solo sistema. Por razones de comparabilidad con los resultados de Lora y Olivera (2007) hemos mantenido las estimaciones por el método de Arellano y Bond.

9 En regresiones que utilizan mínimos cuadrados ordinarios (que no se reportan) hemos comprobado también que aunque los coeficientes pueden estar sesgados, apuntan en la misma dirección que los obtenidos con el método de Arellano y Bond.

10 Aún podría persistir el problema si las series exhiben no estacionaridad en primeras diferencias.

11 En una regresión no incluida en el cuadro encontramos que los países del Este y el Centro de Asia, Europa e incluso Africa incluidos en la muestra (véase la lista de países en el Apéndice 1) gastan significativamente más que América Latina. 


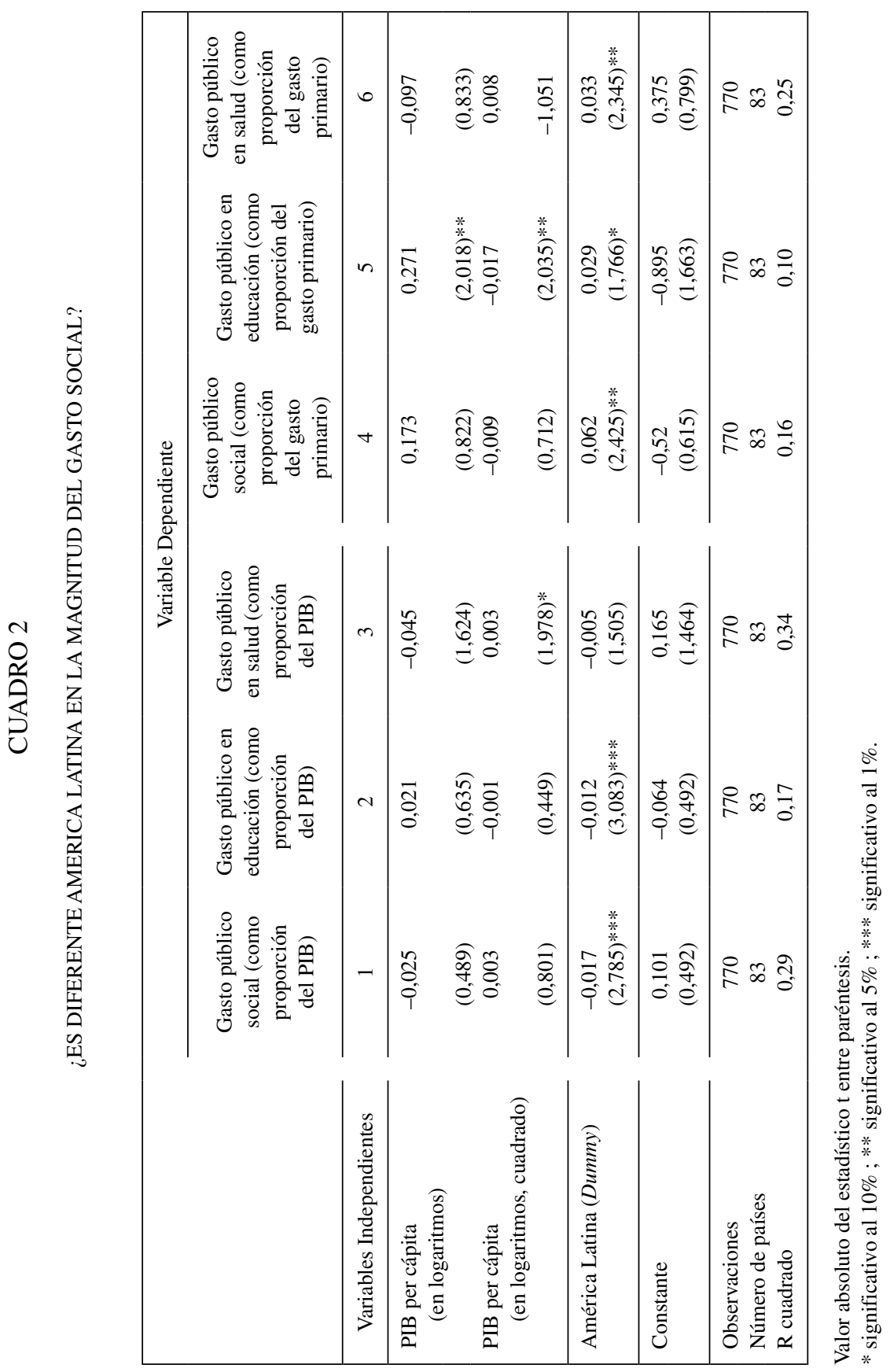


desarrollo. En promedio, en la región se dedica 6,2\% más del presupuesto primario a los sectores sociales, y esta diferencia es significativa al 5\% (regresión 4). De esos 6,2 puntos, 3,3 se deben al sector de salud, y el resto corresponde a educación. Por consiguiente, si los gobiernos latinoamericanos gastan muy poco en los sectores sociales es esencialmente porque el gasto público primario es muy bajo para los patrones mundiales, no porque le asignen poca importancia a las áreas sociales del gasto.

El Cuadro 3 presenta los principales resultados sobre la sensibilidad fiscal del gasto social en el mundo en desarrollo y en América Latina. Según los resultados del primer grupo de variables en la regresión 1, en el mundo en desarrollo el gasto social como proporción del PIB se eleva 13 centavos por cada dólar de aumento del gasto primario, 3 centavos por cada dólar de mayor ingreso fiscal y cerca de 9 centavos por cada dólar de mayores pagos de intereses de la deuda. Sin embargo, si el saldo de la deuda pública (del año anterior) se eleva en un dólar, el gasto social se reduce en 1,3 centavos. Todos estos patrones de reacción del gasto social son estadísticamente significativos al $1 \%$, excepto la respuesta a los ingresos fiscales que sólo es significativa al 10\% (además, el gasto social tiene una cierta inercia propia, que se refleja en el coeficiente de la variable dependiente rezagada).

Antes de discutir en qué aspectos es diferente América Latina, conviene entender las razones de los comportamientos anteriores. No es sorprendente que los gastos sociales se eleven en la medida en que los gobiernos aumentan su gasto primario o reciben mayores ingresos. Obsérvese, sin embargo, que los coeficientes son muy distintos, lo que implica que los gastos sociales pueden reaccionar muy diferente según sea la modalidad de expansión o de ajuste de las cuentas fiscales. Por ejemplo, ante una reducción del déficit fiscal por el $1 \%$ del PIB, típicamente el gasto social se reduce en $0,13 \%$ del PIB cuando el ajuste se basa totalmente en un recorte de gasto, pero aumenta en $0,03 \%$ del PIB si el ajuste se logra totalmente mediante mayores ingresos fiscales. La regresión 2 que utiliza como variable dependiente el gasto social como proporción del gasto público muestra un coeficiente negativo para la variable que mide el gasto primario. Esto significa que el gasto social es más estable que otros gastos y que es menos vulnerable frente a los recortes generales de gasto (consistente con estudios anteriores sobre el tema, reseñados en la introducción). Obsérvese también que, en el mundo en desarrollo en general, no hace mayor diferencia que el aumento del gasto sea por pagos de intereses o por los componentes primarios del gasto (los coeficientes respectivos no son significativamente diferentes). Por último, obsérvese que los aumentos de deuda pública reducen el gasto social (al margen de las decisiones fiscales corrientes que hayan conducido a tales aumentos), tanto en niveles absolutos como también en proporción al gasto público primario total (véase esto último en la regresión 2). Aunque las razones para la mayor sensibilidad de los gastos sociales a los aumentos de deuda deben ser objeto de estudios futuros, pueden avanzarse algunas hipótesis (véase Lora y Olivera, 2007). Los gastos sociales básicos en salud y educación no ofrecen retornos evidentes de corto plazo y -en su componente no salarial- pueden recortarse o aplazarse sin incurrir en costos económicos o políticos elevados en el corto plazo (en cambio, sí hay costos económicos y políticos elevados cuando se aplazan gastos de inversión para obras en curso o gastos corrientes para la prestación de servicios a segmentos más vocales o poderosos, especialmente a los 


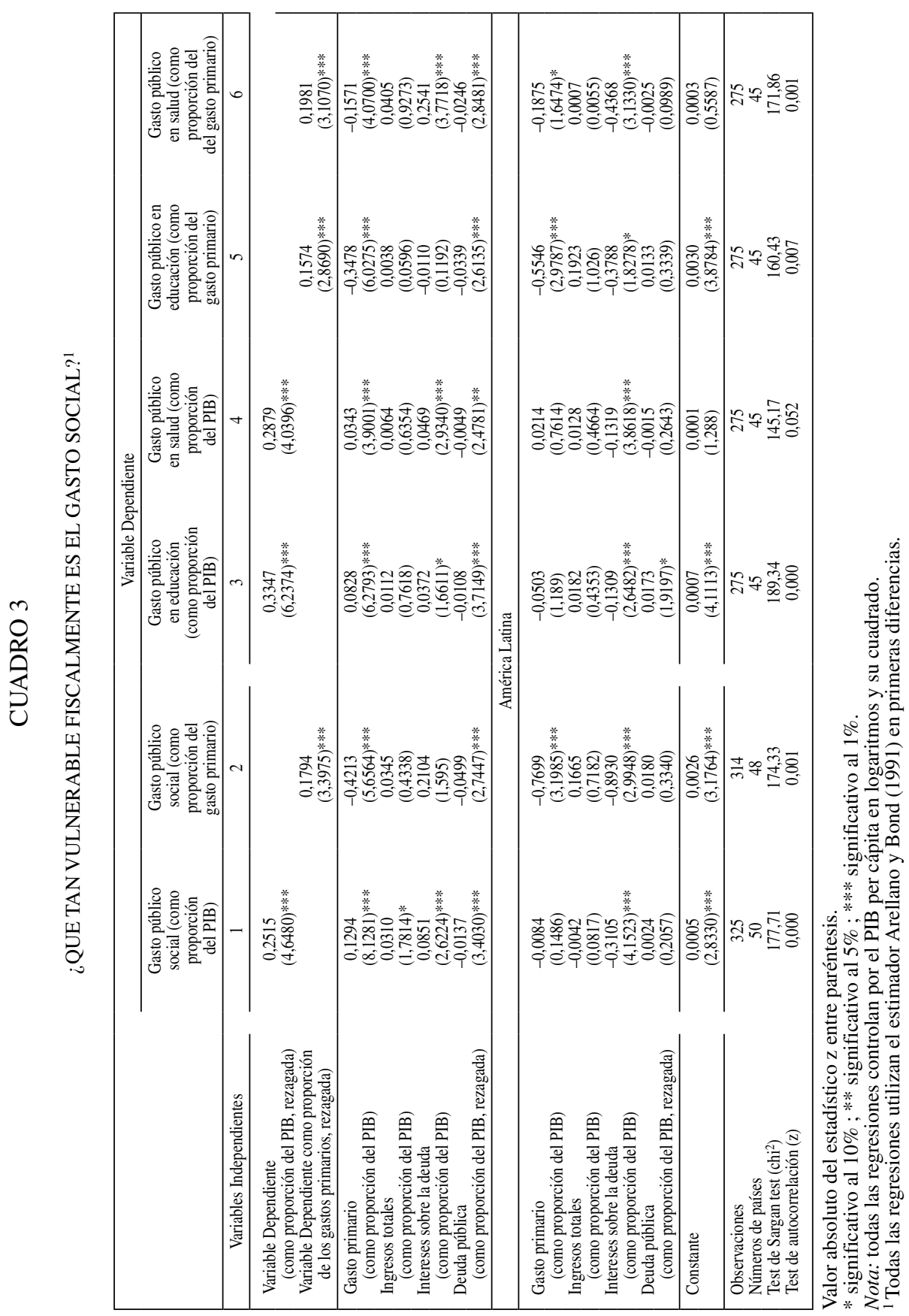


sectores productivos). Puesto que se trata en su mayor parte de gastos recurrentes, usualmente los gobiernos o los congresos no consideran conveniente financiar los gastos sociales con deudas (máxime cuando la madurez de las deudas puede ser más corta que el rendimiento económico o fiscal de tales gastos). En forma consistente con estas hipótesis, Lora (2006) ha encontrado que el gasto público en infraestructura en América Latina aumenta en niveles absolutos y como proporción del gasto público cuando crece la deuda pública (aislando como aquí la influencia de otras variables fiscales).

Los gastos sociales en América Latina difieren de los patrones anteriores en dos aspectos. La diferencia más grande (en significancia estadística y en términos económicos) reside en la respuesta a los pagos de intereses de la deuda. El coeficiente de esta variable para América Latina en la regresión 1 indica un efecto adicional negativo de 31 centavos por cada dólar adicional de pagos de intereses. Esto significa que en vez de aumentar 8 centavos por cada dólar adicional de pagos de intereses, el gasto social en América Latina se reduce 23 centavos. La regresión 2 muestra además que el efecto de desplazamiento relativo del gasto social con respecto al resto de gastos cuando aumentan los pagos de intereses de la deuda es un fenómeno propio de América Latina, que no se observa en el resto del mundo. El otro aspecto en que América Latina es diferente es en la respuesta a los cambios en el gasto primario de la participación del gasto social en el gasto total. Como lo indica el coeficiente respectivo para América Latina en la regresión 2, el gasto social reacciona en forma aún más negativa frente a los aumentos en el gasto primario, y viceversa, lo cual implica una mayor rigidez frente a las fluctuaciones del gasto total. Obsérvese que aunque no es significativo, el coeficiente para esta misma variable en la regresión 1 es negativo y tiene un valor que contrarresta prácticamente el coeficiente positivo de esta variable para el conjunto de todos los países. Lo anterior no significa que el gasto social en América Latina sea insensible a los cambios en la posición fiscal. La rigidez proviene del lado del gasto, no del lado de los ingresos. Cuando éstos aumentan (o disminuyen), el gasto social en América Latina se comporta aproximadamente como en el resto del mundo. De esta forma, resulta válido el argumento de que América Latina necesita recaudar más impuestos si quiere aumentar el gasto social.

Prácticamente todos los comportamientos de respuesta del gasto social a las variables fiscales que hemos identificado hasta ahora, tanto a nivel mundial como para América Latina, son válidos para los dos tipos de gasto social considerados. Sin embargo, la magnitud de las respuestas es diferente en varios aspectos (regresiones 3 a 6 del Cuadro 3). Considerando que los gastos en educación son aproximadamente el doble de los gastos en salud, estos últimos son notablemente más sensibles a los pagos de intereses, tanto en sentido directo en el conjunto del mundo en desarrollo, como en la dirección opuesta entre los países latinoamericanos. También se aprecia una mayor sensibilidad del gasto en salud frente a las fluctuaciones del gasto primario en América Latina: el coeficiente de respuesta total (es decir, sumando los coeficientes correspondientes para el mundo y para América Latina) frente al gasto primario es mayor para salud que para educación en las regresiones 3 y 4 (aunque no son significativos), y sustancialmente más cercano a cero para salud que para educación cuando la variable dependiente es la proporción del gasto primario (regresiones 5 y 6). 


\section{LAS REACCIONES DEL GASTO SOCIAL FRENTE A LAS OPCIONES DE ENDEUDAMIENTO Y DE INCUMPLIMIENTO DE LAS DEUDAS}

Puesto que uno de los resultados más robustos de los ejercicios anteriores es la sensibilidad del gasto social al endeudamiento y a los pagos de intereses de la deuda, ${ }^{12}$ en esta sección nos preguntamos si esos resultados dependen de las opciones de endeudamiento, más específicamente de si los recursos de crédito provienen de fuentes oficiales. Más adelante en esta sección nos preguntamos también si la opción de incumplir las obligaciones de deuda puede tener efectos favorables sobre el gasto social.

En el Cuadro 4 se intenta dar una respuesta a la primera pregunta. Las regresiones 1 y 3 consideran como variables explicativas distintas modalidades de financiamiento, sin incluir otras variables fiscales. De esta forma intentan medir el efecto total del financiamiento sobre el gasto social. El endeudamiento con la banca multilateral (Banco Mundial y bancos oficiales regionales como el Banco Interamericano de Desarrollo o la Corporación Andina de Fomento) tiende a agudizar el efecto negativo del endeudamiento sobre el gasto social. De acuerdo con los coeficientes de la regresión 1, mientras que por cada dólar de endeudamiento el gasto social (en el período siguiente) se reduce 1,5 centavos (y en el largo plazo cerca de 3 centavos, debido al efecto de la variable dependiente rezagada), cuando el endeudamiento ocurre con la banca multilateral ese efecto se eleva en 2,3 centavos (o cerca de 5 centavos en el largo plazo). Nada sugiere que América Latina sea diferente en este respecto. Los coeficientes de la regresión 3 para las variables de deuda oficial para el mundo en desarrollo en su conjunto sugieren que este efecto adicional del endeudamiento multilateral no opera a través de la composición del gasto primario. Si algo ocurre más bien en el caso latinoamericano es que el endeudamiento oficial de tipo bilateral sí eleva la participación del gasto social en el gasto total. En las regresiones 2 y 4 , que incluyen las otras variables fiscales, los coeficientes de las variables de la deuda oficial dejan de ser significativos, tanto para el mundo en desarrollo en general, como para América Latina. Esto sugiere que la influencia de la deuda oficial ocurre a través de estas variables. La comparación de los coeficientes comunes con las regresiones 1 y 2 del Cuadro 3 pone de presente que este efecto ocurre a través del gasto primario. Puesto que al incluir las variables de las deudas oficiales en las regresiones se reduce sensiblemente el coeficiente de esta variable, se deduce que este tipo de endeudamiento lleva a reducir el gasto primario, y que por esta vía tiene un efecto adicional de reducción del gasto social. ${ }^{13}$ Por consiguiente, puede concluirse que el endeudamiento oficial, especialmente con las multilaterales, no ayuda a contrarrestar el efecto

12 En Lora y Olivera (2007) se presentan diversas pruebas adicionales de robustez que confirman estos resultados.

13 Regresiones adicionales, no reportadas, muestran que el gasto primario se reduce en general con los aumentos de deuda (4 centavos por dólar, significativo al 1\%), pero aún más con los aumentos de deuda multilateral (5,8 centavos adicionales, significativo al 10\%) o con el endeudamiento con el FMI (8,4 centavos adicionales, no significativo). En cambio aumenta con la deuda bilateral (5 centavos adicionales, significativo al $5 \%$ ). 


\section{CUADRO 4}

\section{¿AYUDA AL GASTO SOCIAL QUE HAYA FINANCIAMIENTO OFICIAL?}

\begin{tabular}{|c|c|c|c|c|}
\hline \multirow[b]{3}{*}{ Variables Independientes } & \multicolumn{4}{|c|}{ Variable Dependiente } \\
\hline & \multicolumn{2}{|c|}{$\begin{array}{c}\text { Gasto público social } \\
\text { (como proporción del PIB) }\end{array}$} & \multicolumn{2}{|c|}{$\begin{array}{c}\text { Gasto público social } \\
\text { (como proporción del gasto primario) }\end{array}$} \\
\hline & 1 & 2 & 3 & 4 \\
\hline $\begin{array}{l}\text { Variable Dependiente } \\
\text { (como proporción del PIB, rezagada) }\end{array}$ & $\begin{array}{l}0,4666 \\
(7,8463)^{* * * *}\end{array}$ & $\begin{array}{l}0,3520 \\
(6,1937)^{* * * *}\end{array}$ & & \\
\hline $\begin{array}{l}\text { Variable Dependiente } \\
\text { (como proporción de los gastos } \\
\text { primarios, rezagada) }\end{array}$ & & & $\begin{array}{l}0,4382 \\
(6,7745)^{* * *}\end{array}$ & $\begin{array}{l}0,3525 \\
(5,5994)^{* * * *}\end{array}$ \\
\hline Gasto primario & & 0,0867 & & $-0,4257$ \\
\hline Ingresos totales & & 0,0313 & & 0,1204 \\
\hline (como proporción del PIB) & & $(1,356)$ & & $(1,098)$ \\
\hline Intereses sobre la deuda & & 0,0748 & & 0,2135 \\
\hline (como proporción del PIB) & & $(2,4202)^{* *}$ & & $(1,518)$ \\
\hline $\begin{array}{l}\text { Deuda pública } \\
\text { (como proporción del PIB, rezagada) }\end{array}$ & $\begin{array}{l}-0,0155 \\
(3,1934) * * *\end{array}$ & $\begin{array}{l}-0,0198 \\
(4,4565) * * *\end{array}$ & $\begin{array}{l}-0,0513 \\
(2,3348)^{* *}\end{array}$ & $\begin{array}{l}-0,0693 \\
(3,2607)^{* * * *}\end{array}$ \\
\hline $\begin{array}{l}\text { Deuda multilateral } \\
\text { (como proporción del PIB, rezagada) }\end{array}$ & $\begin{array}{l}-0,0230 \\
(2,1453) * *\end{array}$ & $\begin{array}{c}-0,0132 \\
(1,347)\end{array}$ & $\begin{array}{l}-0,0074 \\
(0,1608)\end{array}$ & $\begin{array}{l}-0,0256 \\
(0,5757)\end{array}$ \\
\hline Deuda bilateral & 0,0123 & 0,0131 & $-0,0115$ & 0,0089 \\
\hline (como proporción del PIB, rezagada) & $(1,376)$ & $(1,612)$ & $(0,2939)$ & $(0,2377)$ \\
\hline Deuda con el FMI & $-0,1228$ & $-0,1740$ & $-0,8918$ & $-0,9208$ \\
\hline \multirow[t]{4}{*}{ (como proporción del PIB, rezagada) } & $(0,9729)$ & $(1,411)$ & $(1,614)$ & $(1,6516)^{*}$ \\
\hline & \multicolumn{4}{|c|}{ América Latina } \\
\hline & \multicolumn{4}{|c|}{ Variable Dependiente } \\
\hline & \multicolumn{2}{|c|}{$\begin{array}{c}\text { Gasto público social } \\
\text { (como proporción del PIB) }\end{array}$} & \multicolumn{2}{|c|}{$\begin{array}{c}\text { Gasto público social } \\
\text { (como proporción del gasto primario) }\end{array}$} \\
\hline Variables Independientes & 1 & 2 & 3 & 4 \\
\hline Gasto primario & & 0,0726 & & $-0,4866$ \\
\hline (como proporción del PIB) & & $(0,9453)$ & & $(1,491)$ \\
\hline Ingresos totales & & $-0,0530$ & & $-0,4686$ \\
\hline (como proporción del PIB) & & $(0,8740)$ & & $(1,608)$ \\
\hline Intereses sobre la deuda & & $-0,2493$ & & $-0,8910$ \\
\hline (como proporción del PIB) & & $(3,2351)^{* * *}$ & & $(2,7172)^{* * * *}$ \\
\hline Deuda pública & $-0,0041$ & 0,0359 & 0,0685 & 0,0451 \\
\hline (como proporción del PIB, rezagada) & $(0,3037)$ & $(2,3976)^{* *}$ & $(1,106)$ & $(0,6337)$ \\
\hline Deuda multilateral & 0,0349 & 0,0048 & 0,1049 & 0,1538 \\
\hline como proporción del PIB, rezagada) & $(0,9337)$ & $(0,1333)$ & $(0,5904)$ & $(0,8895)$ \\
\hline Deuda bilateral & 0,0098 & 0,0460 & 0,2494 & 0,0005 \\
\hline (como proporción del PIB, rezagada) & $(0,3839)$ & $(1,635)$ & $(2,4963)^{* *}$ & $(0,0048)$ \\
\hline Deuda con el FMI & 0,0363 & 0,0153 & $-0,0013$ & 0,0627 \\
\hline (como proporción del PIB, rezagada) & $(1,025)$ & $(0,4861)$ & $(0,0084)$ & $(0,4212)$ \\
\hline Constante & $\begin{array}{l}0,0012 \\
(5,0427)^{* * * *}\end{array}$ & $\begin{array}{l}0,0009 \\
(3,7576)^{* * *}\end{array}$ & $\begin{array}{r}0,0017 \\
(1,605)\end{array}$ & $\begin{array}{c}0,0009 \\
(0,7868)\end{array}$ \\
\hline Observaciones & 244 & 244 & 244 & 244 \\
\hline Número de países & 40 & 40 & 40 & 40 \\
\hline
\end{tabular}

Valor absoluto del estadístico z entre paréntesis.

$*$ significativo al $10 \%$; ** significativo al 5\% ; *** significativo al $1 \%$.

Nota: todas las regresiones controlan por el PIB per cápita en logaritmos y su cuadrado.

${ }^{1}$ Todas las regresiones utilizan el estimador Arellano y Bond (1991) en primeras diferencias. 
desfavorable de la deuda sobre el gasto social porque tal tipo de endeudamiento tiende a ir asociado con menor gasto primario. Puede ser que esta asociación se deba a la condicionalidad macro que puede ir vinculada a ese endeudamiento, pero igualmente puede ser que las reducciones de gasto causadas por cualquier razón no captada en las variables explicativas de estos modelos (por ejemplo, ciclos políticos de gasto o dificultades políticas o administrativas para ejecutar el gasto) estén asociadas con la utilización de los recursos de financiamiento oficial.

Finalmente, en el Cuadro 5 se explora el efecto que tienen sobre el gasto social las declaraciones de incumplimiento de las deudas por parte de los gobiernos. Como se observó en la introducción, este es un tema muy central en algunas campañas electorales, que despierta mucho interés público en América Latina, pero sobre el que no hay estudios empíricos (excepto Lora y Olivera, 2007, que es el punto de partida de las estimaciones que siguen). Las variables de interés son ahora una dummy que refleja los eventos de incumplimiento y una variable que interactúa esta dummy con el monto total de la deuda pública vigente. ${ }^{14}$ Las regresiones 1 y 2 replican los resultados de Lora y Olivera (2007) según los cuales los incumplimientos elevan en promedio el gasto social en $0,4 \%$ del PIB y aumentan la participación del gasto social en el gasto primario en 1,7 puntos (en ambos casos con una significancia estadística del 5\%). Sin embargo, la regresión 3 indica que el primero de estos resultados no es robusto a la separación de los países latinoamericanos (ninguno de los coeficientes de incumplimiento es ahora significativo). En cambio, al separar los países latinoamericanos en la regresión 4, que se refiere a la participación del gasto social en el gasto primario, se encuentra que en América Latina los incumplimientos de deuda reducen en promedio la participación del gasto social (el coeficiente negativo obtenido, $-0,1031$, significativo al $1 \%$, más que contrarresta el coeficiente positivo de 0,0475 , significativo al 5\%, para el conjunto de la muestra). El coeficiente de las variables que interactúan la dummy de incumplimiento con el valor de las deudas muestra que en el mundo entero el monto de las deudas no afecta significativamente los resultados, pero en el caso de América Latina sí: sólo en los países que tienen coeficientes de deuda superiores a $95 \%$ del PIB $^{15,16}$ es probable que los incumplimientos aumenten

14 La interacción se calcula como el producto de la dummy y la diferencia entre la deuda pública y el promedio para esta variable para todas las observaciones incluidas en la regresión. La razón para tomar desviaciones del promedio es que, sin alterar el coeficiente del término de interacción, permite interpretar el coeficiente de la variable dummy como el efecto promedio que tienen los incumplimientos (de otra forma, este coeficiente tendría que interpretarse como el efecto del incumplimiento de los países sin deuda, cosa que no tiene sentido).

15 Este cálculo se obtiene como $\mathrm{d}^{*}=\mathrm{dbar}-($ ainc + ainclac $) /\left(\right.$ aincdeuda + aincdeudalac) donde $\mathrm{d}^{*}$ es el coeficiente de deuda en el que el incumplimiento de deuda tiene un efecto neutro sobre el gasto social, dbar es el ratio deuda/PIB promedio de la muestra, ainc es el coeficiente de la variable dummy de incumplimiento de la deuda para el conjunto de países, ainclac es el coeficiente de la variable dummy de incumplimiento de la deuda para América Latina, aincdeuda es el coeficiente de la variable que interactúa la dummy y los ratios de deuda para todos los países, y aincdeudalac es esta última variable para los países latinoamericanos.

16 Hay 20 observaciones en la base de datos utilizada en las que esto ocurre: Argentina 2003; Grenada 2003; Honduras 1991 y entre 1994 y 1996; Jamaica 1986, 1991, 1993, 1994, 1995 y entre 1998 y 2003; Nicaragua 1997; Suriname 1987 y Uruguay 2003. 


\section{CUADRO 5}

¿AYUDA AL GASTO PUBLICO SOCIAL QUE EL GOBIERNO NO PAGUE LA DEUDA?

\begin{tabular}{|c|c|c|c|c|}
\hline & \multicolumn{4}{|c|}{ Variable Dependiente } \\
\hline & $\begin{array}{l}\text { Gasto público } \\
\text { social (como } \\
\text { proporción } \\
\text { del PIB) }\end{array}$ & $\begin{array}{c}\text { Gasto público } \\
\text { social (como } \\
\text { proporción } \\
\text { del gasto } \\
\text { primario) }\end{array}$ & $\begin{array}{l}\text { Gasto público } \\
\text { social (como } \\
\text { proporción } \\
\text { del PIB) }\end{array}$ & $\begin{array}{c}\text { Gasto público } \\
\text { social (como } \\
\text { proporción } \\
\text { del gasto } \\
\text { primario) }\end{array}$ \\
\hline Variables Independientes & 1 & 2 & 3 & 4 \\
\hline $\begin{array}{l}\text { Variable Dependiente } \\
\text { (como proporción del PIB, rezagada) }\end{array}$ & $\begin{array}{l}0,315 \\
(5,801)^{* * * *}\end{array}$ & & $\begin{array}{l}0,245 \\
(4,415)^{* * * *}\end{array}$ & \\
\hline $\begin{array}{l}\text { Variable Dependiente } \\
\text { (como proporción de los gastos primarios, rezagada) }\end{array}$ & & $\begin{array}{l}0,228 \\
(4,352)^{* * * *}\end{array}$ & & $\begin{array}{l}0,148 \\
(2,741)^{* * * *}\end{array}$ \\
\hline Gasto primario (como proporción del PIB) & $\begin{array}{c}0,135 \\
(8,630) * * *\end{array}$ & $\begin{array}{l}-0,542 \\
(6,761) * * *\end{array}$ & $\begin{array}{l}0,132 \\
(8,3560)^{* * *}\end{array}$ & $\begin{array}{l}-0,475 \\
(5,870) * * *\end{array}$ \\
\hline Ingresos totales (como proporción del PIB) & $\begin{array}{l}0,045 \\
(2,725)^{* * *}\end{array}$ & $\begin{array}{c}0,100 \\
(1,160)\end{array}$ & $\begin{array}{l}0,036 \\
(2,077)^{* *}\end{array}$ & $\begin{array}{c}0,105 \\
(1,117)\end{array}$ \\
\hline Intereses sobre la deuda (como proporción del PIB) & $\begin{array}{c}0,017 \\
(0,591)\end{array}$ & $\begin{array}{l}-0,028 \\
(0,235)\end{array}$ & $\begin{array}{l}0,080 \\
(2,480)^{* *}\end{array}$ & $\begin{array}{c}0,159 \\
(1,209)\end{array}$ \\
\hline Deuda pública (como proporción del PIB, rezagada) & $\begin{array}{l}-0,017 \\
(3,911)^{* * *}\end{array}$ & $\begin{array}{l}-0,060 \\
(2,990) * * *\end{array}$ & $\begin{array}{l}-0,016 \\
(3,508)^{* * *}\end{array}$ & $\begin{array}{l}-0,041 \\
(1,907)^{*}\end{array}$ \\
\hline Default (Dummy) & $\begin{array}{l}0,004 \\
(2,204)^{* *}\end{array}$ & $\begin{array}{l}0,017 \\
(2,068) * *\end{array}$ & $\begin{array}{c}0,004 \\
(0,765)\end{array}$ & $\begin{array}{c}0,048 \\
(2,423)^{* *}\end{array}$ \\
\hline \multirow[t]{4}{*}{ Default * Deuda pública } & $\begin{array}{c}0,006 \\
(1,142)\end{array}$ & $\begin{array}{c}0,018 \\
(0,819)\end{array}$ & $\begin{array}{c}0,004 \\
(0,548)\end{array}$ & $\begin{array}{c}-0,049 \\
(1,439)\end{array}$ \\
\hline & \multicolumn{4}{|c|}{ América Latina } \\
\hline & \multicolumn{4}{|c|}{ Variable Dependiente } \\
\hline & $\begin{array}{l}\text { Gasto público } \\
\text { social (como } \\
\text { proporción } \\
\text { del PIB) }\end{array}$ & $\begin{array}{c}\text { Gasto público } \\
\text { social (como } \\
\text { proporción } \\
\text { del gasto } \\
\text { primario) }\end{array}$ & $\begin{array}{l}\text { Gasto público } \\
\text { social (como } \\
\text { proporción } \\
\text { del PIB) }\end{array}$ & $\begin{array}{c}\text { Gasto público } \\
\text { social (como } \\
\text { proporción } \\
\text { del gasto } \\
\text { primario) }\end{array}$ \\
\hline Variables Independientes & 1 & 2 & 3 & 4 \\
\hline Gasto primario (como proporción del PIB) & & & $\begin{array}{c}0,027 \\
(0,471)\end{array}$ & $\begin{array}{l}-0,451 \\
(1,706)^{*}\end{array}$ \\
\hline Ingresos totales (como proporción del PIB) & & & $\begin{array}{c}-0,039 \\
(0,737)\end{array}$ & $\begin{array}{c}0,003 \\
(0,010)\end{array}$ \\
\hline Intereses sobre la deuda (como proporción del PIB) & & & $\begin{array}{l}-0,306 \\
(4,095)^{* * * *}\end{array}$ & $\begin{array}{l}-0,752 \\
(2,543)^{* *}\end{array}$ \\
\hline Deuda pública (como proporción del PIB, rezagada) & & & $\begin{array}{c}-0,002 \\
(0,149)\end{array}$ & $\begin{array}{c}-0,034 \\
(0,579)\end{array}$ \\
\hline Default (Dummy) & & & $\begin{array}{c}-0,003 \\
(0,283)\end{array}$ & $\begin{array}{l}-0,103 \\
(2,697)^{* * * *}\end{array}$ \\
\hline Default * Deuda pública & & & $\begin{array}{c}0,014 \\
(0,962)\end{array}$ & $\begin{array}{l}0,179 \\
(2,823)^{* * *}\end{array}$ \\
\hline Constante & $\begin{array}{l}0,001 \\
(4,739)^{* * * *}\end{array}$ & $\begin{array}{l}0,004 \\
(4,128)^{* * * *}\end{array}$ & $\begin{array}{l}0,001 \\
(3,671)^{* * * *}\end{array}$ & $\begin{array}{l}0,004 \\
(3,249)^{* * * *}\end{array}$ \\
\hline Observaciones & 325 & 275 & 325 & 275 \\
\hline Número de países & 50 & 45 & 50 & 45 \\
\hline
\end{tabular}

Valor absoluto del estadístico z entre paréntesis.

$*$ significativo al $10 \% ; * *$ significativo al $5 \%$; *** significativo al $1 \%$.

Nota: todas las regresiones controlan por el PIB per cápita en logaritmos y su cuadrado.

${ }^{1}$ Todas las regresiones utilizan el estimador Arellano y Bond en primeras diferencias. 
el peso del gasto social en el gasto total, mientras que en los países con menos deuda tiende a ocurrir lo contrario. ${ }^{17}$

\section{CONCLUSIONES E IMPLICACIONES DE POLITICA}

Con base en datos de panel para medio centenar de países en este artículo se analiza la vulnerabilidad del gasto social a las variables fiscales y a las opciones de endeudamiento público en América Latina en comparación con el resto del mundo en desarrollo. En todo el mundo el gasto social responde mucho más fuertemente a las variaciones de los gastos primarios que de los ingresos fiscales. Como es obvio, los ajustes fiscales pueden perjudicar a los gastos sociales cuando no es posible aumentar los ingresos fiscales. Los bajos niveles de tributación -y la dificultad para aumentarlos_en la mayoría de países latinoamericanos implican una limitación severa para el gasto social, que ya ocupa una proporción relativamente elevada del gasto público en la región. Pero América Latina enfrenta una dificultad adicional: a diferencia de lo que ocurre en el resto del mundo, donde los gastos sociales se elevan cuando aumentan los pagos de intereses de la deuda, en los países latinoamericanos hay un fuerte efecto de desplazamiento. En adición, como ocurre en todo el mundo, el gasto social resulta afectado directamente por los mayores niveles de endeudamiento. A pesar de que los organismos multilaterales como el Banco Mundial o el BID orienten parte de sus operaciones de crédito al financiamiento de los sectores sociales, no hay ninguna evidencia de que endeudarse con estos organismos mitigue el efecto adverso de la deuda sobre el gasto social. Antes bien, parece agravarlo, aunque las razones no son claras y no puede descartarse un efecto de causalidad inversa. Para aumentar el gasto social tampoco parece una opción dejar de pagar la deuda. Los incumplimientos de las obligaciones crediticias parecen tener un efecto favorable sobre el gasto social (más exactamente sobre su participación en el gasto total), en el resto del mundo, pero no en América Latina. La evidencia encontrada en este artículo indica que los incumplimientos tienden a reducir el gasto social, excepto en países con ratios de deuda muy elevados (el ratio crítico estimado es 95\% del PIB). Para elevar el gasto social en la región no parece haber otra opción diferente del esfuerzo tributario.

17 Es importante notar que estos resultados se mantienen incluso cuando no se controla por las demás variables fiscales que se incluyen en estas regresiones. Es decir, este efecto no depende de lo que le ocurra a los ingresos fiscales, al gasto primario o a los pagos de intereses. 


\section{REFERENCIAS}

ARELLANO, M. y S. BOND (1991). "Some tests of specification for panel data: Monte Carlo evidence and an application to employment equations", The Review of Economic Studies 58, pp. 277-297.

ARELLANO, M. y O. BOVER (1995). "Another look at the instrumental variable estimation of errorcomponents models", Journal of Econometrics 68, pp. 29-51.

BID (2007). Vivir con Deuda: Cómo Contener los Riesgos del Endeudamiento Público, Progreso Económico y Social en América Latina, Informe 2007, David Rockefeller Center for Latin American Studies, Cambridge (Banco Interamericano de Desarrollo, Washington D.C.).

BAQIR, R. (2002). "Social Sector Spending in a Panel of Countries”, IMF Working Paper WP/02/35.

BLUNDELL, R. y S. BOND (1998). "Initial conditions and moment restrictions in dynamic panel data models", Journal of Econometrics 87, pp. 115-143.

BORENSZTEIN, E. y U. PANIZZA (2006). "The Cost of Default”, IDB Research Department Working Paper WP/08/238.

CEPAL: Comisión Económica para América Latina y el Caribe (2007), Panorama Social. Santiago de Chile.

CHAUVIN, N. y A. KRAAY (2005). "What Has 100 Billion Dollars Worth of Debt Relief Done for Low-Income Countries?", Borrador de trabajo, Universidad de Princeton / Banco Interamericano de Desarrollo / Banco Mundial.

GUPTA, S.; L. DICKS-MIREAUX; R. KHEMANI; C. MCDONALD y M. VERHOEVEN (2000). "Social Issues in IMF-Supported Programs", IMF Occasional Paper 191.

HELLER, P. y J. DIAMOND (1990). "International Comparisons of Government Expenditure Revisited: The Developing Countries 1975-1986”, IMF Occasional Working Paper 69.

HICKS, N. (1989). "Expenditure Reductions in High-Debt Countries", Finance and Development 26, pp. 35-37.

HICKS, N. y A. KUBISCH (1984). "Recent Experience in Cutting Government Expenditures", Finance and Development 21, pp. 37-39.

JAIMOVICH, D. y U. PANIZZA (2006). "Public Debt Around the World: A New Dataset of Central Government Debt”, IDB Research Department Working Paper 561.

LORA, E. (2006). "Public Investment in Infrastructure in Latin America: Is Debt the Culprit?", InterAmerican Development Bank Working Paper 595.

LORA, E. y M. OLIVERA (2007). "Public Debt and Social Expenditure: Friends or Foes?", Emerging Market Review 8 (4), pp. 299-310.

MAHDAVI, S. (2004). "Shifts in the Composition of Government Spending in Response to External Debt Burden", World Development 32 (7), 1139-1157.

MAURO, P. (1998). "Corruption and the Composition of Government Expenditure", Journal of Public Economics 69 (2), pp. 263-279.

PAPAGAPITOS, A. (1992). "The Effects of Stabilization Programs on the Composition of Public Expenditure in Developing Countries: 1972-1988”, disertación doctoral no publicada, Ohio State University.

SNYDER, J. y I. YACKOVLEV (2000). "Political and Economic Determinants of Changes in Government Spending on Social Protection Programs", documento no publicado (Massachusetts Institute of Technology, Cambridge MA). 


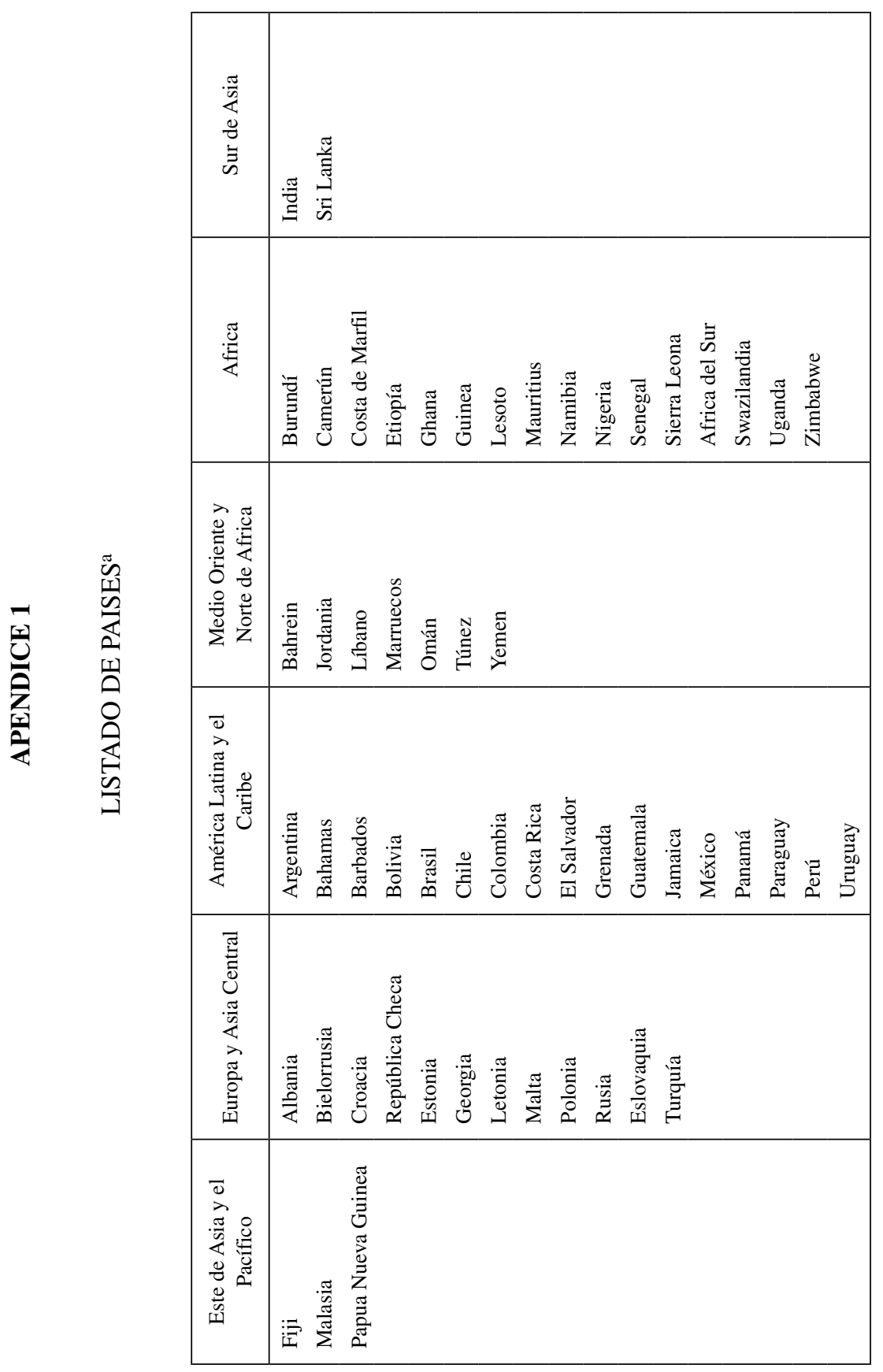

